Ergod. Th. \& Dynam. Sys. (1990), 10, 131-140

Printed in Great Britain

\title{
Attractors in cellular automata
}

\author{
MIKE HURLEY \\ Department of Mathematics and Statistics, Case Western Reserve University, \\ Cleveland, Ohio 44106, USA \\ (Received 5 January 1988)
}

\begin{abstract}
We give a classification theorem for cellular automata, showing that either there is a minimal quasi-attractor whose basin has full measure, or else no chain component has a basin with positive measure.
\end{abstract}

\section{Introduction}

Cellular automata have received much attention in recent years, both from mathematicians and from applied scientists [3]. One reason for this, besides their intrinsic mathematical interest, is that they are believed to provide a class of models for a wide range of physical and biological processes. An aspect of cellular automata that is important for modelling is that certain automata exhibit 'self-organizing behavior'; that is, the property that dissimilar initial conditions tend to similar states under iteration of the automaton. From the dynamical systems point of view this suggests the presence of attracting sets with large basins. The purpose of this paper is to investigate the collection of attractors of a cellular automaton, with the following questions in mind:

(1) What is the hierarchy of these attractors; i.e., how are they ordered under inclusion?

(2) What can be said about the internal structure and dynamics of the attractors that are important in this hierarchy?

The main result of this paper provides a fairly general answer to the first of these questions.

THEOREM. Any cellular automaton $f$ satisfies exactly one of the following:

(1) There is a unique minimal attractor $A$ of $f$. In this case $A$ is contained in every attractor of $f, A$ is shift invariant, and the basin of $A$ is open, dense and has full measure.

(2) There is a unique minimal quasi-attractor $Q$ of $f$ which is not an attractor. In this case $Q$ is contained in every attractor of $f$ and is shift invariant. There are two subcases

(a) the basin of $Q$ has full measure

(b) the basin of any chain component of $f$ (including $Q$ ) has measure zero.

(3) $f$ has a pair of disjoint attractors. In this case $f$ has uncountably many minimal quasi-attractors, and the basin of any chain component of $f$ has measure zero. 
COROllary. In Case 1 or in case 2(a), if the minimal quasi-attractor is a periodic orbit, then it is a single point, fixed by both $f$ and the shift.

The paper is organized as follows: the basic definitions and some background are given in $\S \S 1$ and $2 ; \S 3$ contains the proof of the theorem in the special case of one-dimensional cellular automata; $\$ 4$ describes the generalization to higherdimensional automata; examples and an alternate characterization of the four classes are presented in $\S 5 ; \S 6$ contains a proof of the corollary; and $\S 7$ is a discussion of the relation between the clasification afforded by the theorem and the empirical classification of Wolfram [10,11], and the theoretical classification of Gilman [4]. This paper was largely motivated by the results of Gilman and of Wolfram.

\section{Acknowledgements}

Conversations with Bob Gilman, Mike Boyle and Ethan Coven were helpful in the preparation of this paper.

\section{Cellular automata}

Suppose $S$ is a finite set of symbols, and let $(\Sigma, \sigma)$ denote the (two-sided) shift on $S$. That is, $\Sigma$ is the set of all bi-infinite sequences of elements of $S$, and $\sigma: \Sigma \rightarrow \Sigma$ is given by $(\sigma x)(n)=x(n+1)$.

To avoid confusion we will denote a point in $\Sigma$ as $x$; the $n$th entry in the bisequence $x$ will be denoted $x(n)$, and a sequence of points in $\Sigma$ will be denoted $x_{n}$. Since $\Sigma$ can be thought of as the set of maps from $Z \rightarrow S$, the notation $x(n)$ is natural.

A subbasis for the topology on $\Sigma$ is the collection of cylinder sets. For any fixed $s$ in $S$ and integer $n$, the cylinder set $C(n, s)$ is defined to be $\{x \mid x(n)=s\}$. A metric compatible with this topology is given by $d(x, y)=2^{-i}$ where $i=\min \{|j|: x(j) \neq y(j)\}$. With this topology $\Sigma$ is compact and $\sigma$ is a homeomorphism. If $S=\left\{s_{1}, \ldots, s_{m}\right\}$ and $\left\{p_{i}: 1 \leq i \leq m\right\}$ are strictly positive numbers whose sum is one, then there is a Borel probability measure $\mu$ on $\Sigma$ defined by $\mu\left(\bigcap_{j=1}^{k} C\left(n_{j}, s_{j}\right)\right)=\prod_{j=1}^{k} p_{j}$ whenever the integers $n_{1}, \ldots, n_{k}$ are distinct. As is well known, $\mu$ is $\sigma$-invariant and the system $(\Sigma, \sigma, \mu)$ is strongly mixing. In particular if $U, V$ are subsets of $\Sigma$ of positive measure, then there is an $N$ such that $\sigma^{n}(U) \cap V$ has positive measure for all $n \geq N$ [2].

A one dimensional cellular automaton is a map $f: \Sigma \rightarrow \Sigma$ that commutes with $\sigma$. A characterization of these maps was discovered by Curtis et al. [5]: $f$ commutes with $\sigma$ if and only if there is an integer $k$ and a map $f_{0}: S^{2 k+1} \rightarrow S$ such that for each $x$ in $\Sigma$ and each integer $n,(f(x))(n)=f_{0}(x(n-k), \ldots, x(n+k)) . f_{0}$ is called a block map that generates $f$.

Higher dimensional cellular automata are defined similarly. Let $\Sigma_{n}$ denote the set of maps from the integer lattice $Z^{n}$ to $S$, so if $x \in \Sigma_{n}$ and $\vec{p} \in Z^{n}$, then $x(\vec{p}) \in S$. Let $G$ be the group of automorphisms of $\Sigma_{n}$ that is generated by shifts in the individual coordinates. That is, $g \in G$ if and only if there is $\vec{q} \in Z^{n}$ with the property that $(g x)(\vec{p})=x(\overrightarrow{p+q})$ for all $\vec{p} \in Z^{n}$. In this case we will write $g=g_{q}$. This gives a natural isomorphism between $G$ and $Z^{n}$. An $n$-dimensional cellular automaton is a 
map $f: \Sigma_{n} \rightarrow \Sigma_{n}$ that commutes with each $g \in G$. The proof of the Curtis-HedlundLyndon theorem in [5] generalizes directly to the $n$-dimensional case. To make the meaning of this precise, for each $m \geq 0$ consider the finite subset $[-m, m]^{n}$ of $Z^{n}$; call this subset $I(m)$. Let $J(m)$ denote the set of maps from $I(m)$ to $S$. A block map is any map from $J(m)$ to $S$. The block map $f_{0}$ generates a cellular automaton $f$ by $(f x)(\vec{p})=f_{0}\left(T_{m}\left(g_{-\vec{p}}(x)\right)\right)$, where $T_{m}: \Sigma_{n} \rightarrow J(m)$ is the truncation operator $\left(T_{m} x\right)(q)=x(q)$ for any $\vec{q} \in I(m)$. The Curtis-Hedlund-Lyndon theorem says that any cellular automaton is generated by a block map. There are $G$-invariant ergodic probability measures on $\Sigma_{n}$; their definition is analogous to the one-dimensional case. All statements about measure on $\Sigma_{n}$ refer to such a measure.

To keep the argument as simple as possible, we will prove the theorem first in the one-dimensional case. The extension to higher dimensional cellular automata is not hard, and is discussed in $\$ 4$.

\section{Attractors and chain recurrence}

The definition of attractor that we will use is that of C. Conley. Suppose $X$ is a compact metric space and $f: X \rightarrow X$ is continuous. A closed nonempty subset $A$ of $X$ is an attractor for $f$ if there is an open neighbourhood $U$ of $A$ with $f(\operatorname{clos}(U)) \subset U$ and $A=\bigcap_{n \geq 0} f^{n}(U)$. In this situation the basin of attraction of $A$ is the open set $B(A)=\bigcup_{n \geq 0} f^{-n}(U)$. This is a weak definition in that it imposes no structure on the dynamics of $f$ on $A$; for instance the set $Y=\bigcap_{n \geq 0} f^{n}(X)$ is an attractor for $f$ (let $U=X$ ). We choose to work with this definition because of the connection between the chain recurrent behaviour of $f$ and the hierarchy of the attractors of $f$. Before describing this connection, we obtain the following preliminary results.

LEMMA 2.1. The number of attractors of $f$ on $X$ is at most countable.

Proof. Let $\mathcal{U}$ be a countable basis of $X$, and let $\mathscr{V}$ be all finite unions of sets in $\mathcal{U}$, so $\mathscr{V}$ is also countable. Let $\mathscr{A}$ be the set of attractors of $f$. By compactness, for each $A \in \mathscr{A}$ there is a set $U(A) \in \mathscr{V}$ with $A \subset U(A)$ and $\operatorname{clos}(U(A)) \subset B(A)$. This gives an injection $\mathscr{A} \rightarrow \mathscr{V}$.

LEMMA 2.2. Suppose $A$ is an attractor of $f$, that $G$ is an open neighbourhood of $A$, and that $K$ is a compact subset of $B(A)$. Then there is a positive integer $n$ with $f^{n}(K) \subset G$. Proof. By hypothesis there is an open set $U$ with $f(\operatorname{clos}(U)) \subset U, \bigcup_{n \geq 0} f^{-n}(U)=$ $B(A)$, and $\bigcap_{n \geq 0} f^{n}(U)=A$. By compactness of $K$, there is a positive integer $m$ such that

$$
K \subset \bigcup_{n=0}^{m} f^{-n}(U)=f^{-m}(U) .
$$

Similarly there is a positive integer $l$ with

$$
f^{\prime}(\operatorname{clos}(U))=\bigcap_{n=0}^{l} f^{n}(\cos (U)) \subset G .
$$

Thus $f^{m+1}(K) \subset G$.

Results similar to the next lemma, but in more specialized settings, can be found in $[1,6,9]$. 
LEMMA 2.3. If $G$ is any neighbourhood of an attractor $A$, then there is an open subset $V$ of $G$ satisfying

(i) $f(\operatorname{clos}(V)) \subset V$, and

(ii) $\bigcap_{n \geq 0} f^{n}(V)=A$,

Proof. Since $A$ is an attractor, there is an open set $U \subset X$ satisfying (i) and (ii). Without loss of generality we can assume $G \subset U$, so that any neighbourhood of $A$ in $G$ will satisfy (ii). To obtain (i), observe first that, because of compactness, there is an open set $W$ with $A \subset W \subset U$ and

$$
\text { (*) } f^{n}(\operatorname{clos}(W)) \subset G \text { for all } n \geq 0 \text {. }
$$

Since $W \subset U$, Lemma 2.2 ensures that there is an integer $N \geq 1$ with $f^{N}(\operatorname{clos}(W)) \subset$ $W$. Now, for $\delta>0$ define open sets $V_{1}, \ldots, V_{N+1}$ as follows (the notation suppresses the dependence of $V_{j}$ on $\delta$ ). Let $V_{1}=W$, and let $V_{j+1}$ be the $\delta$-neighbourhood of $f\left(V_{j}\right)$. Note that as $\delta$ decreases to $0, V_{j+1}$ shrinks to $f^{j}(W)$. It follows from (*) that for sufficiently small $\delta>0$ the open set $V=\bigcup_{j=1}^{N} V_{j}$ is contained in $G$. We finish by verifying that $V$ satisfies (i) as long as $\delta$ is small enough:

$$
f(\operatorname{clos}(V))=\bigcup_{j=1}^{N} f\left(\operatorname{clos}\left(V_{j}\right)\right) \subset \bigcup_{j=1}^{N} V_{j+1} \subset V \cup V_{N+1} \text {. }
$$

$V_{N+1}$ shrinks to $f^{N}(W)$ as $\delta$ decreases to 0 , and $N$ was chosen so that

$$
f^{N}(\operatorname{clos}(W))=\operatorname{clos}\left(f^{N}(W)\right) \subset W \subset V, \text { so that } V_{N+1} \subset V \text { for all small } \delta>0 \text {. }
$$

Next we outline the relationship between chain recurrence and the hierarchy of attractors. The basic results are due to $\mathrm{C}$. Conley [1]; other treatments in contexts closer to that of this paper can be found in $[6,7]$.

An $\varepsilon$-chain for $f$ is a (finite or infinite) sequence of points in $X,\left(x_{i}\right)$, such that $d\left(f\left(x_{i}\right), x_{i+1}\right)<\varepsilon$ for all possible values of $i$. When the $\varepsilon$-chain is finite, say $0 \leq i \leq n(n>0)$, we will say that the $\varepsilon$-chain goes from $x_{0}$ to $x_{n}$. A point $x$ is chain recurrent if there is an $\varepsilon$-chain from $x$ to $x$ for each $\varepsilon>0$. Let $C R(f)$ denote the set of chain recurrent points of $f$. Conley's basic result is the following.

Proposition 2.4. (Conley, [1, p. 37].) $X-C R(f)=\bigcup[B(A)-A]$, where the union is taken over all of the attractors $A$ of $f$.

We will not present the full proof of 2.4 , but the idea behind it is simple. It is easy to check that if $x \in B(A)-A$, then $x$ is not in $C R(f)$ (use 2.3). Conversely, if $x$ is not chain recurrent, then there is an $\varepsilon>0$ such that $x \notin U$ where $U$ is the set $\{y \in X \mid$ there is an $\varepsilon$-chain from $\omega(x)$ to $y\}(\omega(x)$ denotes the omega-limit set of $x)$. One can show [7, Lemma 2.3] that $U$ is open, and $f(\operatorname{clos}(U)) \subset U$. Thus $A=\bigcap_{n \geq 0} f^{n}(U)$ is an attractor with $x \in B(A)-A$.

There is a natural equivalence relation on $C R(f)$ given by $x \sim y$ if and only if for each $\varepsilon<0$ there are $\varepsilon$-chains going from $x$ to $y$ and from $y$ to $x$. The equivalence classes are closed subsets of $X$ called the chain components of $f$. A chain component $C$ has the property that it contained in any attractor whose basin intersects $C$. If $x$ is any point of $X$, then its omega limit set is contained in a unique chain component 
$C$. Let $B(C)$ denote the set of $x$ such that $C$ contains $\omega(x)$, and call this set the basin of $C$. The collection of basins of chain components of $f$ forms a partition of $X$ in a dynamically natural way.

The chain components that we will be most concerned with are the minimal quasi-attractors of $f$. A nonempty subset $Q$ of $X$ is a quasi-attractor of $f$ if $Q=\bigcap_{n \geq 0} A_{n}$, where the $A_{n}$ are attractors of $f . Q$ is actually an attractor of $f$ only if $Q$ can be realized as the intersection of a finite number of attractors. $Q$ is a minimal quasi-attractor if no proper subset of $Q$ is also a quasi-attractor. (If a minimal quasi-attractor is an attractor, we will simply call it a minimal attractor.) It is easy to see that any quasi-attractor contains a minimal quasi-attractor, and it follows from 2.4 that any minimal quasi-attractor is a chain component.

The following simple results are listed for reference.

LEMMA. 2.5. If $Q_{1}$ and $Q_{2}$ are disjoint quasi-attractors of $f$, then there are disjoint attractors of $f, A_{1}$ and $A_{2}$, with $Q_{i} \subset A_{i}, i=1,2$.

Proof. Use the fact that any nonempty finite intersection of attractors in an attractor to see that anytime $Q$ is a quasi-attractor, then there is a sequence of attractors, each containing $Q$, and with the sequence converging to $Q$ in the Hausdorff topology on closed nonempty subsets of $X$.

LEMMA 2.6. (i) If $C, C^{\prime}$ are chain components whose basins intersect, then $C=C^{\prime}$ (ii) If the basins of two attractors intersect, then so do the attractors.

Proof. (i) If $x$ is in the intersection of the basins, then $\omega(x) \subset C \cap C^{\prime}$; if two chain components intersect, they must be equal. The proof of (ii) is similar.

LEMMA 2.7. Suppose $\sigma: X \rightarrow X$ is a homeomorphism commuting with $f$.

(i) If $A$ is an attractor of $f$, then so is $\sigma(A)$, and $B(\sigma(A))=\sigma B(A)$

(ii) If $C$ is a chain component of $f$, then so is $\sigma(C)$, and $B(\sigma(C))=\sigma B(C)$.

The proof is trivial.

3. Proof of the theorem in the one-dimensional case

Assume that $f: \Sigma \rightarrow \Sigma$ is a one-dimensional cellular automaton, and $\sigma$ is the shift.

Proposition 3.1. Suppose $f$ has 2 disjoint attractors, $A$ and $A^{\prime}$. Then any attractor of $f$ contains a pair of disjoint attractors, and consequently $f$ has uncountably many minimal quasi-attractors.

Proof. Let $A^{\prime \prime}$ be any attractor of $f$. Since basins of attractors are open and $\sigma$ is mixing, there is an $n$ such that the sets $B\left(\sigma^{n}(A)\right) \cap B\left(A^{\prime \prime}\right)$ and $B\left(\sigma^{n}\left(A^{\prime}\right)\right) \cap B\left(A^{\prime \prime}\right)$ are nonempty. Thus by $2.6 \sigma^{n}(A) \cap A^{\prime \prime}$ and $\sigma^{n}\left(A^{\prime}\right) \cap A^{\prime \prime}$ are attractors contained in $A^{\prime \prime}$. They are disjoint since $\sigma$ is a homeomorphism. Proceeding inductively, one sees that for any sequence of 0 's and 1's there is a nested sequence of attractors, and the intersection of the attractors in one sequence is disjoint from the corresponding intersection for a different sequence of 0's and 1's. Each one of these intersections of attractors is a quasi-attractor, and therefore contains a minimal quasi-attractor. Thus there are as many minimal quasi-attractors as there are sequences of 0's and 1 's. 
LEMMA 3.2. If $C$ is a chain component of $f$, then either $\sigma^{n}(C) \cap C=\varnothing$ for all $n \neq 0$, or else there is an $n>0$ with $\sigma^{n}(C)=C$.

Proof. Chain components are either equal to disjoint.

Proposition 3.3. Suppose $C$ is a chain component of $f$ and $\mu(B(C))>0$. Then $C$ is a minimal quasi-attractor. Moreover, $\mu(B(C))=1$, every attractor of $f$ contains $C$, and $C$ is fixed by $\sigma$.

Proof. $\mu$ is $\sigma$-invariant so by 3.2 there must be a least positive integer $k$ with $\sigma^{k}(C)=C$. We claim that $k=1$. To see this note that since $\sigma$ is mixing, it follows that $\sigma^{k n}(B(C))$ and $B(\sigma(C))$ intersect for all sufficiently large $n$. By 2.6 and the fact that $C$ is fixed by $\sigma^{k}$, it follows that $C=\sigma(C)$. Now $B(C)$ is $\sigma$-invariant and has nonzero measure, so by ergodicity $\mu(B(C))=1$. If $A$ were an attractor of $f$ that does not contain $C$, then $B(A)$ would be in the complement of $B(C)$; since $B(A)$ is open, this is impossible. Thus $C$ lies in the intersection of all the attractors of $f$. This intersection is clearly a minimal quasi-attractor and therefore is a chain component. It follows that $C$ is equal to this minimal quasi-attractor.

Now we can establish the one-dimensional version of the theorem. 3.1 and 3.3 show that if $f$ has a pair of disjoint attractors, then Case 3 of the theorem holds. Hence we can assume that all of the attractors of $f$ intersect, and so by 2.5 there is a unique minimal quasi-attractor $Q$. Because of this uniqueness, $Q$ is $\sigma$-invariant. If $Q$ is actually an attractor, then its basin has positive measure, and 3.3 implies case 1 of the theorem. If $Q$ is not an attractor, then 3.3 implies case 2 .

\section{Proof of the theorem in the general case}

In order for the proof given in the previous section to carry over the $n$-dimensional case, all that is needed is a verification that the shifts on $\Sigma_{n}$ are mixing. Let $\sigma_{1}: \Sigma_{n} \rightarrow \Sigma_{n}$ denote the shift in the first coordinate; that is $\left(\sigma_{1}(x)\right)\left(p_{1}, p_{2}, \ldots, p_{n}\right)=$ $x\left(p_{1}+1, p_{2}, \ldots, p_{n}\right)$.

Proposition 4.1. Suppose $V, W$ are subsets of $\Sigma_{n}$ of positive measure. Then is an integer $M$ with the property that if $m \geq M$, then $\mu\left(\sigma_{1}^{m}(V) \cap W\right)>0$.

Proof. Let $\varepsilon=\mu(V) \cdot \mu(W) / 3$. Since $\mu$ is a regular measure, there are open and closed sets $\hat{V}, \hat{W}$ with $V \subset \hat{V}, W \subset \hat{W}, \mu(\hat{V}-V)<\varepsilon$, and $\mu(\hat{W}-W)<\varepsilon$. Since open and closed sets in $\Sigma_{n}$ are defined by restricting the values of $x(\vec{p})$ for some finite set of lattice points $\bar{p} \in Z^{n}$, there is an $M$ such that $m \geq M$ implies that

$$
\mu\left(\sigma_{1}^{m}(\hat{V}) \cap \hat{W}\right)=\mu\left(\sigma_{1}^{m}(\hat{V})\right) \cdot \mu(\hat{W})=\mu(\hat{V}) \mu(\hat{W}) \geq \mu(V) \mu(W)=3 \varepsilon .
$$

But

$$
\begin{aligned}
\mu\left(\sigma_{1}^{m}(\hat{V}) \cap \hat{W}\right) & \leq \mu\left(\sigma_{1}^{m}(V) \cap W\right)+\mu\left(\sigma_{1}^{m}(V) \cap(\hat{W}-W)\right)+\mu\left(\sigma_{1}^{m}(\hat{V}-V) \cap \hat{W}\right) \\
& \leq \mu\left(\sigma_{1}^{m}(V) \cap W\right)+2 \varepsilon .
\end{aligned}
$$

Thus $\mu\left(\sigma_{1}^{m}(V) \cap W\right) \geq \varepsilon>0$. 


\section{Examples and characterization}

In this section we give examples to show that Cases 1, 2(a), and 3 of the theorem are realized. We do not know of any examples of case $2(\mathrm{~b})$. It is easy to find examples of Cases 1 and 3. For instance, the zero map $(f x)(j)=0$ for all $x$ and all $j$ is in case 1 , and the identity map is in Case 3 (since $\Sigma$ is a Cantor set, the chain components of the identity map are just the individual points of $\Sigma$ ). Next we give an example of Case 2(a).

Consider the cellular automaton $f$ generated by the block map

$$
\begin{array}{llll}
000 \rightarrow 0 & 001 \rightarrow 0 & 010 \rightarrow 0 & 011 \rightarrow 1 \\
100 \rightarrow 0 & 101 \rightarrow 0 & 110 \rightarrow 0 & 111 \rightarrow 1 .
\end{array}
$$

For each $k$ with $\infty>k \geq-\infty$, let $x_{k}$ be defined by $x_{k}(n)=1$ if and only if $n>k$. Note that each $x_{k}$ is fixed by $f$ and that the sequence $x_{k}$ converges to $z$, where $z$ denotes the bisequence consisting entirely of zeroes. Also for $y \in \Sigma$ we have

5.1. if $y(n)=0$ for arbitrary large values of $n$, then $z$ is the omega limit set of $y$.

5.2. if $y(k)=0$ and $y(n)=1$ for all $n>k$, then $x_{k}$ is the omega limit set of $y$.

It follows that the chain components of $f$ are its individual fixed points. It is clear from 5.1 that $\mu(B(z))=1$. Since any neighborhood of $z$ contains points whose omega limit sets are not equal to $\{z\},\{z\}$ is not an attractor. This rules out Cases $1,2(\mathrm{~b})$, and 3 of the theorem.

It is obvious that if $f$ has only finitely many attractors, then $f$ is an example of Case 1. Additionally, Case 1 can occur when $f$ has an infinite number of attractors. For example, consider the one-dimensional automaton with symbols $\{0,1,2\}$ that is generated by the following block map $f_{0}$. Define $f_{0}(a, b, c)=b$ if none of $a, b, c$ is equal to 2, and $f_{0}(a, b, c)=2$ otherwise. Let $B=\{x \in \Sigma \mid x(n) \in\{0,1\}$ for all $n\}$; note that each $x \in B$ is a chain component of $f$. The proof of 2.4 shows that if $C, C^{\prime}$ are distinct chain components, then there is an attractor $A$ that contains $C$ and is disjoint from $C^{\prime}$. It follows that for $f$ to have an infinite number of chain components it must have infinitely many attractors, and so $f$ is not an example of Case 1 of the theorem. On the other hand it is clear that the fixed point $x$ defined by $x(n)=2$ for all $n$ is a minimal attractor whose basin has full measure.

As with any clasification theorem, there is the practical problem of determining the classification of specific examples. The following observations provide some information concerning this problem.

LеммA 5.3. If $A$ is an attractor of $f$, then there is an open and closed set $C$ with $A \subset C \subset B(A)$ and $f(C) \subset C$.

Proof. The collection of open and closed sets of $\Sigma_{n}$ form a basis for its topology. Since $A$ is compact and $B(A)$ is open there is an open and closed set $K$ with $A \subset K \subset B(A)$. Using 2.2, there is an $m>0$ with $f^{m}(K) \subset K$. Let $C=\bigcup_{0}^{m-1} f^{j}(K)$.

Let $\mathscr{C}=\left\{C \subset \Sigma_{n} \mid C\right.$ is nonempty, open, closed, and $\left.f(C) \subset C\right\}$. If $C \in \mathscr{C}$, then $\bigcap_{n \geq 0} f^{n}(C)$ is an attractor $A_{c}$ whose basin is $\bigcup_{n \geq 0} f^{-n}(C)$. 
LEMMA 5.4. $f$ satisfies Case 3 of the theorem if and only if there is $a C \in \mathscr{C}$ and $n \in Z$ with $\sigma^{n}(C) \cap C=\varnothing$.

Proof. The existence of such a $C$ obviously implies Case 3. Conversely, let $A^{\prime}, A^{\prime \prime}$ be disjoint attractors of $f$. Let $C^{\prime}, C^{\prime \prime}$ be open and closed sets with $A^{\prime} \subset C^{\prime} \subset B\left(A^{\prime}\right)$, $A^{\prime \prime} \subset C^{\prime \prime} \subset B\left(A^{\prime \prime}\right)$. Choose $n$ such that $\sigma^{-n}\left(C^{\prime}\right) \cap C^{\prime \prime} \neq \varnothing$. Let $C=\sigma^{-n}\left(C^{\prime}\right) \cap C^{\prime \prime}$.

Proposition 5.5. Assume that $\sigma^{n}(C) \cap C \neq \varnothing$ for all $C \in \mathscr{C}$ and $n \in Z$.

(a) $f$ is an example of Case 2(b) of the theorem if and only if there is a $C \in \mathscr{C}$ with $\mu\left(B\left(A_{c}\right)\right)<1$.

(b) Assume in addition that $\mu\left(B\left(A_{c}\right)\right)=1$ for all $C \in \mathscr{C}$. Consider the condition (*):

(*) There is a $C \in \mathscr{C}$ such that if $C^{\prime} \in \mathscr{C}$, then there is an $n>0$ with $f^{n}(C) \subset C^{\prime}$. With these hypotheses, $f$ satisfies Case 1 of the theorem if and only if $(*)$ holds, and $f$ satisfies Case $2(a)$ if and only if (*) fails.

Proof. (a) Cases 1 and 2(a) of the theorem require every attractor to have a basin of full measure. Conversely, if $Q$ is a minimal quasi-attractor whose basin has measure 0 , then there is a nested sequence of attractors, $A_{n+1} \subset A_{n}$, with $Q=\bigcap A_{n}$. Then $B(Q)=\bigcap B\left(A_{n}\right)$, so if $\mu(B(Q))$ has measure 0 , then $\mu\left(B\left(A_{n}\right)\right)<1$ for sufficiently large $n$, and the result follows from 5.3.

(b) If Case 1 of the theorem holds let $A$ be the minimal attractor. Note that every $C \in \mathscr{C}$ contains $A$. Choose $C \in \mathscr{C}$ such that $C \in B(A)$. Since any $C^{\prime} \in \mathscr{C}$ is a neighbourhood of $A, 2.2$ implies that (*) holds. If case $2(\mathrm{a})$ of the theorem holds, let $Q$ be the minimal quasi-attractor. For any $C \in \mathscr{C}, Q \varsubsetneqq A_{c}$ and we can find an attractor $A^{\prime}$ with $Q \subset A^{\prime} \subsetneq A_{c}$. Use 5.3 to find $C^{\prime} \in \mathscr{C}$ with $A^{\prime} \subset C^{\prime} \subset B\left(A^{\prime}\right)$. If $f^{n}(C) \subset C^{\prime}$ for any $n$, then

$$
A_{c}=\bigcap_{n \geq 0} f^{n}(C) \subset \bigcap_{n \geq 0} f^{n}\left(C^{\prime}\right)=A^{\prime},
$$

which is absurd, so (*) must fail.

Of course the implementation of 5.4 and 5.5 can be quite difficult. It is no small problem to determine $\mathscr{C}$. However, there has been some effort directed towards describing open and closed invariant sets of one dimensional automata [8].

\section{Periodic attractors}

In this section we will establish the following.

Proposition 6.1. Suppose $Q$ is minimal quasi-attractor of $f$ with $\mu(B(Q))=1$. Then $Q$ is a minimal quasi-attractor of $f^{n}$ for each $n>1$.

Corollary. If, in the proposition, $Q$ is a periodic orbit of $f$, then $Q$ must be a single fixed point, $p$, with $\sigma(p)=p$ (that is, $p: Z^{n} \rightarrow S$ is a constant map).

The corollary follows directly from the proposition, for if the periodic orbit had period $k$, then it would consist of $k$ chain components for $f^{k}$. Thus $Q$ is a single point, which is fixed by $\sigma$ because $Q$ is. We use the following lemma in the proof of 6.1 . 
LEMMA 6.2. If $C$ is a chain component of $f$ and $n \geq 1$, then $C$ consists of the disjoint union of at most $n$ chain components of $f^{n}$.

Proof. Let $n \geq 1$ and $\varepsilon>0$ be given. By the uniform continuity of $f$, there is a $\delta>0$ such that if $\left(x_{i}\right)$ is a $\delta$-chain for $f$, then $\left(x_{n i}\right)$ is an $\varepsilon$-chain for $f^{n}$. Pick some point $q \in C$. We claim that the union of the chain components of $f^{n}$ containing $f^{j}(q)$, $0 \leq j<n$, contains $C$. To see this, let $y$ be a point of $C$, so that there are finite $\delta$-chains for $f$ from $y$ to $q$ and from $f^{n-1} q$ to $y$. Consequently there is a periodic $\delta$-chain for $f,\left(x_{i}\right)$, say of period $m$, with $x_{0}=x_{m}=y$ and $x_{k+j}=f^{j}(q)$ for some $k$ satisfying $0 \leq k \leq m-n+1$ and all $j$ with $0 \leq j \leq n-1$. Now $\left(x_{i n}\right)$ is a periodic $\varepsilon$-chain for $f^{n}$ containing $y$ and one of the points $f^{j}(q), 0 \leq j<n$. Since $\varepsilon>0$ was arbitrary and the set $\left\{f^{j}(q) \mid 0 \leq j<n\right\}$ is finite, the claim follows. It is easy to see that any chain component of $f^{n}$ lies inside a single chain component of $f$, so the proof of the lemma is complete.

Proof of 6.1. Suppose $Q$ is the disjoint union of $C_{1}, \ldots, C_{k}$ where each $C_{j}$ is a chain component of $f^{n}$. The sets $C_{j}$ are closed, pairwise disjoint, and invariant under $f^{n}$, so there are pairwise disjoint closed neighbourhoods $U_{j}$ of $C_{j}$ satisfying:

(a) $f^{n}\left(U_{j}\right) \cap U_{i} \neq \varnothing$ if and only if $i=j$.

Define $U=\bigcup_{j=1}^{k} U_{j}$, and let $A$ be an attractor of $f$ with $Q \subset A \subset U$ (as in the proof of Lemma 2.5) Use Lemma 2.3 to obtain an open neighbourhood $V$ of $A$ with $V \subset U$ and

(b) $f(\operatorname{clos}(V)) \subset V$.

Set $V_{j}=V \cap U_{j}$. It follows from (a), (b) that $f^{n}\left(\operatorname{clos}\left(V_{j}\right)\right) \subset V_{j}$. Define $A_{j}=$ $\bigcap_{m \geq 0} f^{n m}\left(V_{j}\right)$, so $A_{j}$ is an attractor for $f^{n}$. Let $\omega\left(x, f^{n}\right)$ denote the omega-limit set of $x$ under $f^{n}$. If $k>1$, then the attractors $A_{1}, A_{2}$ of $f^{n}$ are disjoint, and the theorem applied to $f^{n}$ implies that the basin of each of its chain components has measure 0 . In particular, each of the sets $W_{j}=\left\{x \mid \omega\left(x, f^{n}\right) \subset C_{j}\right\}, 1 \leq j \leq k$, has measure 0 and consequently $W=\bigcup_{1}^{k} W_{j}$ also has measure 0 . Since an omega-limit set is contained in a single chain component, we see that $W=\left\{x \mid \omega\left(x, f^{n}\right) \subset Q\right\}$. But $\omega\left(x, f^{n}\right) \subset \omega(x, f)$, so this last equality implies that $W$ contains $\{x \mid \omega(x, f) \subset Q\}$. This last set is $B(Q)$, which is assumed to have measure 1 . Thus $W$ cannot have measure 0 , and so $k=1$.

\section{Relation to other classifications}

There are at least two classification schemes for cellular automata other than the one presented in this paper. R. H. Gilman in [4] shows that for a one-dimensional cellular automata $f$, either there is a closed, $f$-invariant set $Y \subset \Sigma$ of almost full measure on which the iterates of $f$ act equicontinuously, or else $f$ resembles an expansive map in that there is an $\varepsilon>0$ such for any $x \in \Sigma$, the set $\left\{y \in \Sigma \mid d\left(f^{n}(x), f^{n}(y)\right)<\varepsilon\right.$ for all $\left.n=0,1, \ldots\right\}$ has measure 0 . Gilman's results provided one of the main motivations for the current investigation. The problem of relating Gilman's classification to the classification given by the theorem is open. A conjecture that seems plausible is that Gilman's equicontinuous case occurs if and only if $f$ has an attractor $A$, not necessarily minimal, whose basin has full measure, and on which $f$ is an almost periodic map. 
Another classification scheme, based on extensive numerical experimentation, has been presented by S. Wolfram. The classification is based on qualitative characterization of long-term iterative behaviour of random initial conditions $[10,11]$

(1) tends to a constant map $x: Z^{n} \rightarrow S$.

(2) localized periodic structures appear and persist.

(3) chaotic behavior.

(4) complicated localized structures, sometimes long-lived.

A possible description of the relation of Wolfram's classes to the theorem is as follows.

Class 1: attracting fixed point.

Class 2: Case 2 of the theorem; the persistence of localized periodic structures suggests the existence of disjoint, invariant, open and closed sets.

Class 3: Cases 1 or 2(a) of the theorem, where the dynamics of $f$ on the minimal quasi-attractor are chaotic.

Class 4: Case 2 of the theorem, where the minimal quasi-attractor is a fixed point. The typical existence of long-lived localized structures might be explained in terms of $f$-invariant sets that are close to the minimal quasi-attractor.

\section{REFERENCES}

[1] C. Conley. Isolated Invariant Sets and the Morse Index. Amer. Math. Soc.: Providence, 1978.

[2] M. Denker, C. Grillenberger \& K. Sigmund. Ergodic Theory on Compact Spaces. Springer Lect. Notes in Math., 527 Springer-Verlag: New York: 1976.

[3] D. Farmer, T. Toffoli \& S. Wolfram. Eds., Cellular automata: Proc. of an interdisciplinary workshop. Physica 10D $(1,2)(1984)$.

[4] R. Gilman. Classes of linear automata. Ergod. Th. \& Dynam. Sys. 7 (1987), 105-118; also, Periodic behavior of linear automata. pp. 216-219 in: Dynamical Systems. Lecture Notes in Mathematics 1342. Springer Verlag, New York, 1988.

[5] G. Hedlund. Endomorphisms and automorphisms of the shift dynamical system. Math. Sys. Th. 3 (1969), 320-375.

[6] M. Hurley. Attractors: persistence, and density of their basins, Trans. Amer. Math. Soc. 269 (1982), 247-271.

[7] M. Hurley, Generic properties of attractors in Euclidean spaces. Proc. of the Berkeley-Ames Conference on Nonlinear Problems in Control and Fluid Dynamics, pp. 283-298. L. Hunt \& C. Martin, eds. Math. Sci. Press: Brookline, Mass., 1984.

[8] E. Jen. Invariant strings and pattern-recognizing properties of one-dimensional cellular automata. J. Stat. Phys $\mathbf{4 3}$ (1986), 243-265.

[9] J. Milnor. On the concept of attractor: correction and remarks. Commun. Math. Phys. 102 (1985), 517-519.

[10] S. Wolfram. Statistical mechanics of cellular automata. Rev. Mod. Phys. 55 (1983), 601-644.

[11] S. Wolfram. Universality and complexity in cellular automata. Physica 10D (1984), 1-35. 